Scripta METALLURGICA et MATERIALIA
Vol. 24, pp. $251-256,1990$

Printed in the U.S.A.
Pergamon Press plc

All rights reserved

\title{
INFLUENCE OF ANISOTROPIC VIBRATIONAL MOTION ON DIFFRACTION FROM GRAIN BOUNDARIES
}

\author{
R. Najafabadi ${ }^{+}$, D. J. Srolovitz ${ }^{+}$, and R. LeSar* \\ +Department of Materials Science and Engincering, University of Michigan \\ Ann Arbor, MI 48109 USA \\ *Theoretical Division, Los Alamos National Laboratory \\ Los Alamos, NM 87545 USA \\ (Received October 23, 1989) \\ (Revised November 28, 1989)

\section{INTRODUCTION}

$X$-ray diffraction has been used extensively, in recent years, to analyze the structure of high angle grain boundaries (1-4). A major difficulty in the interpretation of the $X$-ray results involves correcting for the vibrational motion of the atoms in the boundary (the Debye-Waller factor). Common practice is to assume that all of the atoms in the grain boundary have the same vibrational amplitude, independent of their local environment (1-4). Recent simulations (5) show that the magnitude and the direction of the atomic vibrations vary from atom to atom in the grain boundary region, suggesting that the assumption of isotropic vibrations is not valid. The experimental scattering factor is generally deconvoluted assuming isotropic vibrations in order to determine the atomic structure of the grain boundary. Fitzsimmons and Sass (3) have attempted to take the assymetric nature of the vibrations into account by fitting their scattering data with two vibrational frequencies (parallel and perpendicular to the grain boundary). In the present paper, we demonstrate that the assumptions of uniform and isotropic vibrations (one or two frequencies) does not sufficiently take the highly inhomogeneous environment of a grain boundary into account and could lead to marked changes in the experimentally derived grain boundary structure.

We have recently introduced an approximate method for calculating the free energy of defects in solids, which we called the local-harmonic (LH) model (6). In this method, the vibrations of each atom are determined by calculating the local-dynamical matrix (ignoring the coupling of vibrations of different atoms) and are employed in a determination of the free energy within the framework of an Einstein model. The structure of a defect is calculated by minimizing the free energy with respect to the atomic coordinates. The vibrational frequencies of each atom thus reflect the local atomic distribution around the atom at finite temperature.

In this paper, we calculate the scattering intensities from $\Sigma=5$ and $\Sigma=13(001)$ twist boundaries in gold based upon atomic structure determined from finite temperature atomic relaxations that include the vibrational distributions found within the LH model (6). We show that the assumption of isotropic, homogeneous atomic vibrations leads to predicted scattering factors that differ greatly from those obtained with the true anisotropic, inhomogeneous vibrations.

\section{THEORY}

The integrated $X$-ray scattering intensity is given by $C_{0}<F_{h k l}\left(\left.T\right|^{2}>\right.$, where (hkl) is the reflecting plane and $C_{0}$ is a constant that includes information about the Lorentz polarization, beam absorption, illuminated area, resolution, atomic scartering factor, etc. and will be ignored hereafter. The effects of thermal vibration are included in the finite-temperature structure factor, Fhll(T), which is defined as

$$
F_{h k l}(T)=\sum_{i=1}^{N} \exp \left[2 \pi i\left(\vec{R}_{i}+\vec{r}_{i}\right) \cdot \vec{K}\right]
$$

where $r_{i}$ is the displacement of atom $i$ from the averaged position $R_{i}$ and $K$ is the vector normal to the reflecting planes. Thus, the vibrational distribution around each atomic site is needed to calculate $F_{\text {hkl. }}$ 
In the LH model, the average position and three normal mode frequencies are determined for each atom as a function of temperature. The potential energy of an atom vibrating about its equilibrium position is given in the harmonic approximation by:

$$
E_{i}=\frac{1}{2} m\left[\left(r_{x, i} \omega_{x, i}\right)^{2}+\left(r_{y, i} \omega_{y, i}\right)^{2}+\left(r_{z, i} \omega_{z, i}\right)^{2}\right]
$$

where $m$ is the mass of the atom and where $\left(r_{x}, i, r_{y}, i\right.$, and $\left.r_{z, i}\right)$ and $\left(\omega_{x, i}, \omega_{y, i}\right.$, and $\left.\omega_{z}, i\right)$ are the displacements and vibrational frequencies of atom $i$ (the force constant for the hamonic vibration of atom $i$ in the $x$ direction is $m \omega_{x} i^{2}$ ), respectively, expressed in its normal mode coordinates. The scattering intensity, $I_{h k l}=\left\langle\left|F_{h k l}(T)\right|^{2}\right\rangle$, can be found by averaging over these independent atomic vibrations to find

$$
I_{h k 1}=\left\langle\left|F_{h k 1}(T)\right|^{2}\right\rangle=\left|\sum_{i=1}^{N} \exp \left[-2 \pi^{2}\left\langle r_{i}^{2}\right\rangle / \alpha_{h k l}^{2}\right] \exp \left[2 \pi i \vec{R}_{i} \cdot \vec{K}\right]\right|^{2}
$$

where $d_{h k l}$ is the interplaner spacing and $\left\langle\sigma_{i}^{2}\right\rangle_{K}$ is the mean square displacement of atom $i$ along the $K$ direction. In the LH model, the mean-square displacement can be expressed by:

$$
\left(H_{i}^{2}\right)_{k}=\frac{k_{B} T}{m}\left[\left(K_{x} / \omega_{x, i}\right)^{2}+\left(K_{y} / \omega_{y, j}\right)^{2}+\left(K_{z} / \omega_{z, i}\right)^{2}\right] d_{h k l}^{2}
$$

where $K_{x}, K_{y}$, and $K_{z}$ are components of vector $K$ in the normal mode coordinates of atom $i$.

For a system where all atoms are assumed to have the same isotropic environment the mean-square displacements of all atoms are the same such that Eq. (3) reduces to the Debye-Waller form for the scattering intensity, IDW,

$$
I_{h k l}^{D W}=\exp \left[-4 \pi^{2} B(T) / d_{h k l}^{2}\right]\left|\sum_{i=1}^{N} \exp \left[2 \pi i \vec{R}_{i} \cdot \vec{K}\right]\right|^{2}
$$

where $B(T)=\left\langle r^{2}\right\rangle$ is the mean-square displacement of each atom. This equation is simply the Debye-Waller factor multiplied by the static-scattering factor, $\mathrm{I}$,

$$
I_{\text {hkl }}^{o}=\left|\sum_{i=1}^{N} \exp \left[2 \pi i \overrightarrow{R_{i}} \cdot \vec{K}\right]\right|^{2}
$$

Since the vibrational properties for each atom in a defect system are different, one introduces errors in predicting the integrated intensities of the scattering by simply using the same distribution for each atom. We can quantify this difference by defining an effective mean-square displacement $\left(B_{\text {eff }}\right)$ for the atoms in a grain boundary as a function of scattering direction by calculating the ratio of the scattering intensity (including all vibrations) to the static-scattering intensity,

$$
\frac{I_{h k l}}{P_{h k l}^{0}}=\exp \left[-4 \pi^{2} B_{e f f}(T)_{K} / d_{h k l}^{2}\right]
$$

Any dependence of $B_{e f f}(T)_{K}$ on the scattering direction is an indication that there is anisotropy in the atomic distributions.

\section{RESULTS}

The LH model (6) was used to determine the relaxed structures (as a function of temperature) of two [001] twist grain boundaries in gold with reciprocal densities of coincident sites $(\Sigma)$ of 5 and 13 and misorientation angles of 36.9 and 22.6 degrees, respectively. Periodic boundary conditions were employed in the directions parallel to the grain boundary and perfect crystal blocks were used at the edges of the simulation cell in the direction perpendicular to the grain boundary. Relative translations of the two subcrystals were allowed in the directions parallel and perpendicular to the block such that the pressure within the simulation cell was always zero. The embedded-atom method (EAM) gold potential of Foiles, Baskes, and Daw (7), was used to represent the atomic interactions. A more complete discussion of the minimization procedure as well as details about the structure and thermodynamic properties of these boundaries will be presented elsewhere (9). 
The scattering intensities, $I_{h k l}$, were calculated as a function of temperature using the equilibrium structures and vibrational frequencies found within the LH model (6). Room-temperature values of Ihtl for several reflecting planes are given in Table I for the $\Sigma=5$ boundary and Table II for $\Sigma=13$. Also shown in Tables I and II are results for the staticscattering factor [ $\left[1^{\circ}\right.$, Eq. (6)] and the Debye-Waller model [ $[\mathrm{DW}$, Eq. (5)] for the same structures. For the Debye-Waller model, we used the value for $B(T)\left(=1.08 \AA^{2} / 8 \pi^{2}\right)$ employed in an analysis of the experimental $X$-ray scattering studies of the $\Sigma=13$ grain boundary in gold (2). The percent deviations between the intensities calculated with the LH frequencies and with the Debye-Waller factor are also given. These deviations range from 8 to $47 \%$ and from 6 to $23 \%$ for the $\Sigma=5$ and $\Sigma=13$ boundaries, respectively.

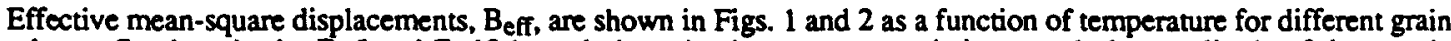
boundary reflections in the $\Sigma=5$ and $\Sigma=13$ boundaries. As the temperature is increased, the amplitude of the atomic vibrations increase. Correspondingly, the deviation between the mean square atomic displacements $B_{\text {eff }}$ for the different reflections becomes more pronounced. This deviation increases with temperature in a roughly linear manner. For the $\Sigma=5$ boundary, the slopes vary between $1.3 \times 10^{-5}$ and $4.5 \times 10^{-5} \AA^{2} / 0 \mathrm{~K}$ for the $[7,3,0]$ and $[4,1,0]$ planes, respectively. The slopes for $\Sigma=13$ calculated from Figure 2 vary between $1.9 \times 10^{-5}$ and $5.5 \times 10^{-5} \AA^{2} / 0 \mathrm{~K}$ for the $[4,0,0]$ and $[10,0,0]$ planes, respectively. This range of slopes for the $\Sigma=13$ boundary can be compared to the value of $3.9 \pm 0.08 \times 10^{-5}$ $\AA^{2} / \mathrm{K}$ found in the analysis of experimental scattering data using the Debye-Waller model (8).

Since the grain boundary structure is not periodic along the direction normal to the interface plane, the reflections are modulated rods of intensity called relrods. The calculated intensity profile as a function of 1 (the Miller index perpendicular to the boundary plane) for the $[4,0]$ relrod is plotted in Figure 3 for the $\Sigma=5$ boundary. We have also plotted the same profile calculated using the Debye-Waller correction term with $B(T)=1.08 \AA^{2} / 8 \pi^{2}$. Use of a constant Debye-Waller correction leads to an overestimate of intensities in the range of $1=[-2.5,-2.1]$, whereas it underestimates the intensity by as much as $10 \%$ in the ranges $[-2.1,0.5]$. One should note that the error for a $[4,0]$ reflection point is only $9.4 \%$; thus, the difference in the two calculated intensity profiles is not as large as it would be for other relrods, such as the $[5,1]$ where the error is approximately $50 \%$. Although the deviations between the Debye-Waller and the full vibrational spectrum intensities are of order $10 \%$, comparison with the static structure factor (no vibrations) shows that the Debye-Waller correction to the static structure factor overestimates the true correction by nearly $50 \%$.

\section{DISCUSSIONAND CONCLUSIONS}

The large deviations in Tables I and II between the intensities calculated with the local-harmonic model frequency distribution, $I_{h k l}$, and those calculated with the constant $B$ Debye-Waller factor, $I_{h k l} D W$, illustrate the sensitive dependence of the scattering intensity on the description of the atomic vibrational distributions. That sensitivity is also clearly seen in the relrods [Fig. (3)], where substantial errors are found when the experimental mean-square displacement is used in the Debye-Waller factor. While another choice of the Debye-Waller factor (i.e. with $B_{e f f}$, or for that matter two different Debye-Waller factors (one along the direction normal to the boundary and another in the plane of the boundary) as used in the case of $\Sigma=13$ boundary (3), might improve agreement between calculated relrods for a specific scattering direction, the strong dependence of $\mathbf{B}_{\text {eff }}$ on scattering direction seen in Figures 1 and 2 indicates that no single value of $B_{\text {eff }}$ can adequately describe all scattering data.

It is difficult to say what errors are introduced into an experimentally derived atomic structure when the constant B Debye-Waller factor approximation is used. The general procedure used in analyzing scattering data is to calculate the static scattering intensity, $\mathrm{I}_{\mathrm{hkl}}{ }^{\mathrm{o}}$, from the experimental data by assuming $\mathrm{B}$ is constant and using Eq. (5). This static scattering intensity is then compared to calculated intensities based on some static model structure. In some cases, the model structure is modified until agreement is reached. If a constant Debye-Waller factor is used, then the $I_{h k 1^{\circ}}$ calculated from the experimental data is in error. We can estimate that error in the present results from our data in Tables I and II. If we assume that $\mathrm{I}_{\mathrm{hkl}}$ is the correct result for the scattering intensity, then the static scattering intensity would be given by $I_{h k V} / \exp \left[-4 \pi^{2} \mathrm{~B} / \mathrm{d}_{h k l^{2}}\right]$ if $\mathrm{B}$ were assumed constant. Comparison of that estimate of $\mathrm{I}_{\text {hkl }}{ }^{\circ}$ with the actual values for $I_{h k l}{ }^{\circ}$ show errors roughly comparable to those between $I_{h k l}$ and $I_{h k l} D W$. Thus, since the errors are different for various scattering directions, it is inevitable that structures determined from the incorrect static scattering will be in error. However, it is not possible to say at this point how severe the error would be.

The origin of these discrepancies can be understood by examining Figure 4 where the distribution of vibrational frequencies of atoms in the boundary calculated with the LH model for the $\sum=5$ boundary at room temperature is plotted [the magnitude of the mean-square displacements is related to the frequency by Eq. (4)]. The frequency distribution is extends over roughly $\pm 20 \% \omega_{0}$, where $\omega_{0}$ is the vibrational frequency of an atom in the perfecet crystal. The large spread of frequencies is an indication that the environment around the atoms in the boundary is very inhomogeneous, and that to assume a single, isotropic vibrational frequency is not a good approximation. A more complete discussion of the distribution of vibrational motion will be given elsewhere (9).

Unfortunately, it is not possible to experimentally measure an effective mean-square displacement as a function of scattering direction. The most promising approach is probably to include theoretical calculations of the mean-square 
displacements in the data analysis. These distributions might be determined in a number of ways, e.g. molecular dynamics, Monte Carlo calculations (5), or approximate methods such as used here.

Acknowledgement - This research was supported by the Division of Materials Science of the Office of Basic Energy Sciences of the U. S. Department of Energy, Grant No. FG02-88ER-45367.

\section{BEEERENCES}

1. J. Budai, P. D. Bristowe, and S. L. Sass, Acta Metall. 31, 699 (1976).

2. M. R. Fitzsimmons and S. L. Sass, Acta Metall. 36, 3103 (1988).

3. M. R. Fitzsimmons and S. L. Sass, Acta Metall. 37, 1009 (1989).

4. I. Majid, P. D. Bristowe, and R. W. Balluffi, Phys. Rev. B 40, 2779 (1989).

5. S. M. Foiles, Acta Metall. 37, 2815 (1989)

6. R. LeSar, R. Najafabadi, and D. J. Srolovitz, Phys, Rev. Lett. 63, 624 (1989).

7. S. M. Foiles, M. I. Baskes, and M. S. Daw, Phs. Rev. B 33, 7983 (1986).

8. M. R. Fitzsimmons, E. Burkel, and S. L. Sass Phys. Rev. Lett. 61,2237 (1988).

9. R. Najafabadi, D. J. Srolovitz, and R. LeSar, to be publised.

TABLE I

Calculated Room-Temperature Scartering Intensities for the $\Sigma=5$ Twist Boundary in Gold.*

\begin{tabular}{llrrrr} 
h & $\mathbf{k}$ & $\mathrm{I}_{\text {hkl }}$ & \multicolumn{1}{c}{$\mathrm{I}_{\mathbf{h k l}}{ }^{0}$} & \multicolumn{1}{c}{$\mathrm{I}_{\text {hkl }} \mathrm{DW}$} & $\Delta \%$ \\
& & & & & \\
3 & 3 & 65.232 & 76.022 & 60.305 & -7.6 \\
1 & 1 & 0.363 & 0.399 & 0.389 & 7.1 \\
2 & 0 & 5.025 & 5.595 & 5.314 & 5.7 \\
2 & 2 & 33.724 & 35.010 & 31.585 & -6.3 \\
3 & 2 & 3.455 & 3.971 & 3.360 & -2.8 \\
4 & 0 & 54.914 & 61.128 & 49.755 & -9.4 \\
4 & 1 & 15.296 & 18.840 & 15.138 & -1.0 \\
5 & 1 & 5.040 & 3.740 & 2.676 & -46.9 \\
5 & 2 & 4.667 & 5.731 & 3.946 & -15.4 \\
5 & 3 & 4.885 & 5.470 & 3.532 & -27.7 \\
5 & 4 & 31.827 & 41.088 & 24.244 & -23.8 \\
6 & 1 & 30.082 & 38.041 & 23.631 & -21.4 \\
7 & 2 & 11.909 & 14.264 & 7.212 & -39.4 \\
7 & 3 & 8.194 & 9.919 & 4.703 & -42.6
\end{tabular}

TABLE II

Calculated Room-Temperature Scattering Intensities for the $\Sigma=13$ Twist Boundary in Gold.*

$\begin{array}{rrrrrr}\text { h } & \text { k } & I_{\text {hkl }} & I_{h k l^{0}} & I_{h k l} D W & \Delta \% \\ 5 & 5 & 806.577 & 967.845 & 755.687 & -6.3 \\ 10 & 6 & 319.686 & 525.572 & 268.121 & -16.1 \\ 10 & 4 & 409.213 & 597.698 & 336.640 & -17.7 \\ 6 & 0 & 329.574 & 377.544 & 315.932 & -4.1 \\ 9 & 3 & 309.233 & 372.274 & 238.467 & -22.9 \\ 7 & 3 & 137.642 & 170.338 & 127.836 & -7.1 \\ 9 & 1 & 138.424 & 164.846 & 109.860 & -20.6 \\ 4 & 0 & 142.073 & 146.602 & 135.441 & -4.7 \\ 6 & 5 & 162.213 & 191.437 & 141.553 & -12.7 \\ 10 & 0 & 40.271 & 69.910 & 42.620 & 5.8 \\ 10 & 1 & 121.005 & 165.276 & 100.262 & -17.1 \\ 10 & 5 & 23.982 & 39.045 & 21.033 & -12.3 \\ 4 & 2 & 72.972 & 75.416 & 68.309 & -6.4 \\ 9 & 5 & 33.531 & 48.994 & 28.995 & -13.5 \\ 8 & 7 & 20.653 & 32.294 & 18.461 & -10.6 \\ 11 & 5 & 34.561 & 64.614 & 31.371 & -9.2\end{array}$

* The intensities of the [hk] reflections calculated (arbitrary unit) with the local-harmonic model vibrational frequencies ( $I_{h k l}$ ), assuming no vibrational motion $\left(I_{h k l}{ }^{\circ}\right.$ ), and assuming a uniform, isotropic, vibration $\left(I_{h k l}{ }^{D W}\right)$. $\Delta \%$ is the percent deviation between $I_{h k l}$ and $I_{h k l} D W$. 


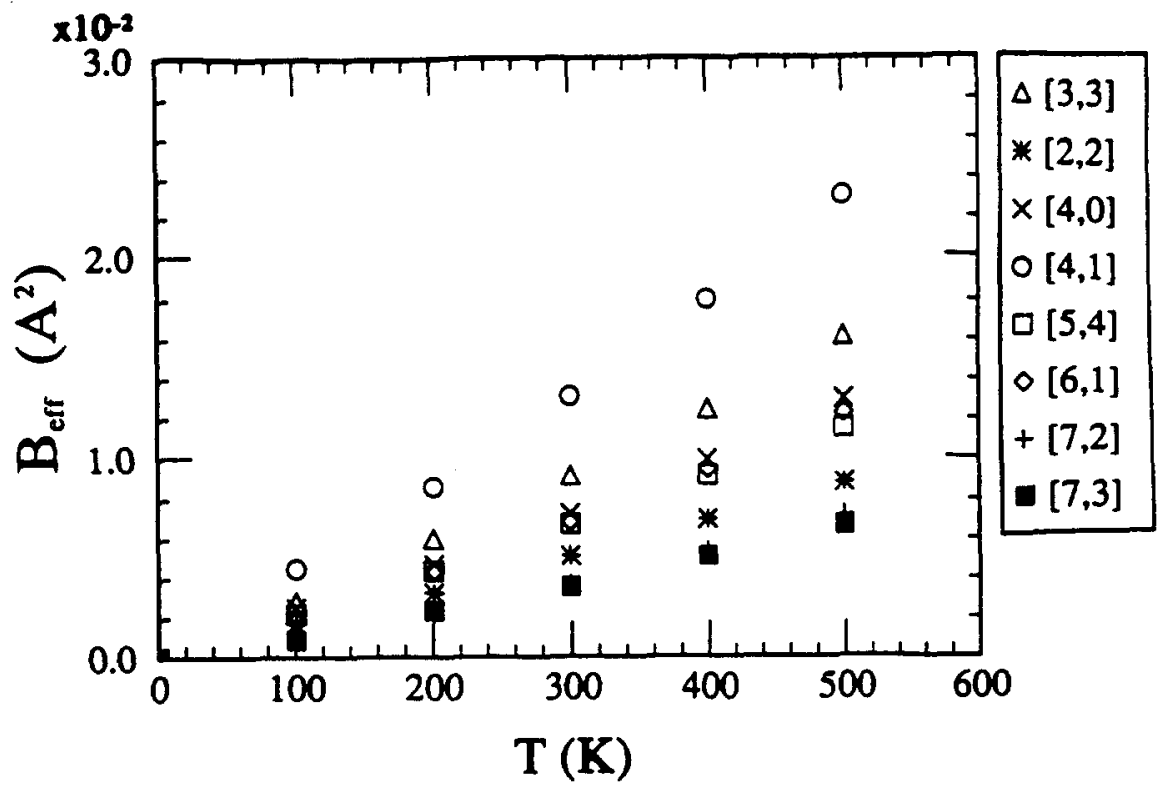

Figure 1. Calculated values of the effective mean-square radius, $B_{\text {eff }}(T) K$ (Eq. 7), as a function of temperature for a $\Sigma=5$ twist boundary in gold. The various symbols indicate different scattering planes.

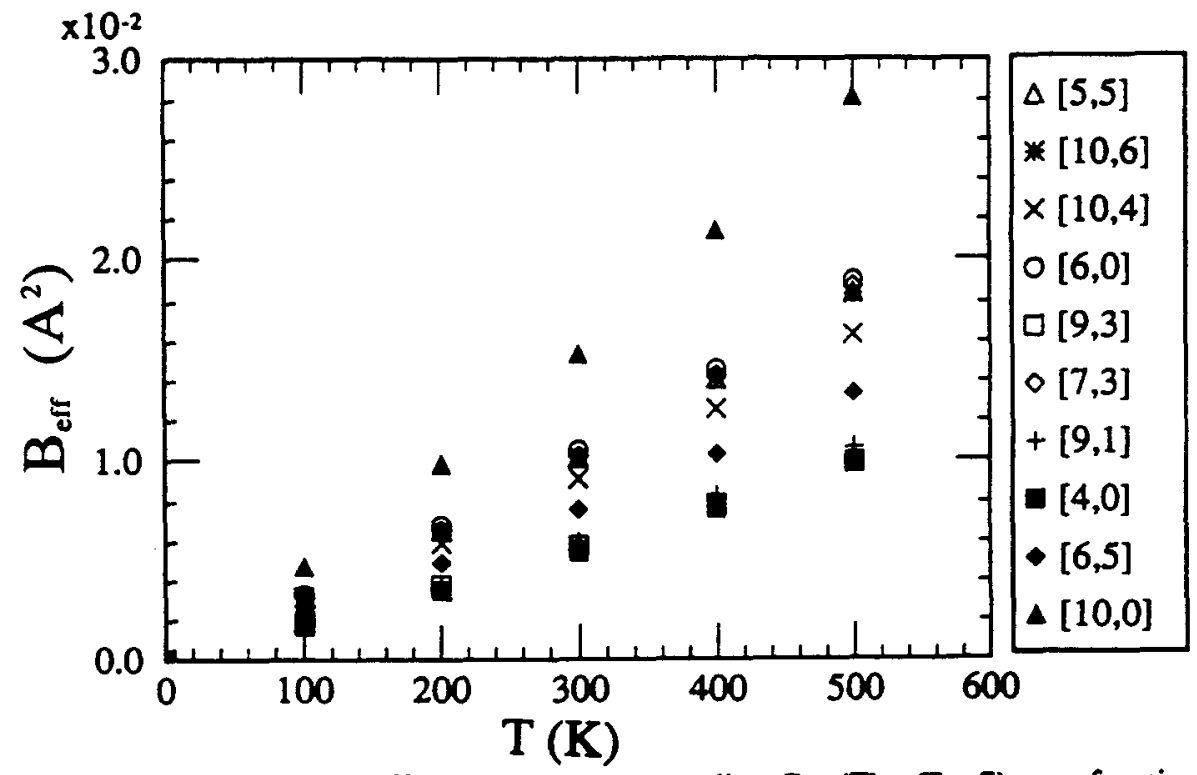

Figure 2. Calculated values of the effective mean-square radius, $B_{\text {eff }}(T)_{K}(E q .7)$, as a function of temperature for a $\Sigma=13$ twist boundary in gold. The various symbols indicate different scattering planes 


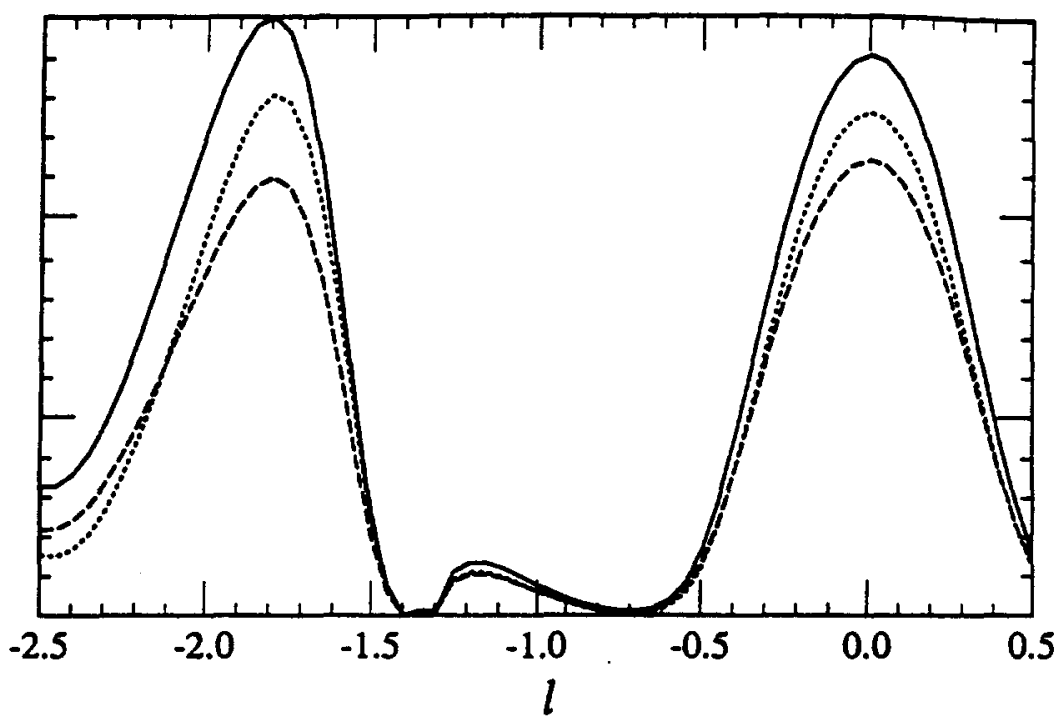

Figure 3. Calculated scattering intensity (arbitrary unit) of the $[4,0]$ relrod in a $\Sigma=5$ twist boundary in gold as a function of the miller index, 1 , perpendicular to the boundary. The dotted curve was calculated using the local-harmonic model vibrational distribution the dashed curve assumed that all atoms had the same, isotropic, atomic distribution, and the solid curve assumed no thermal vibration.

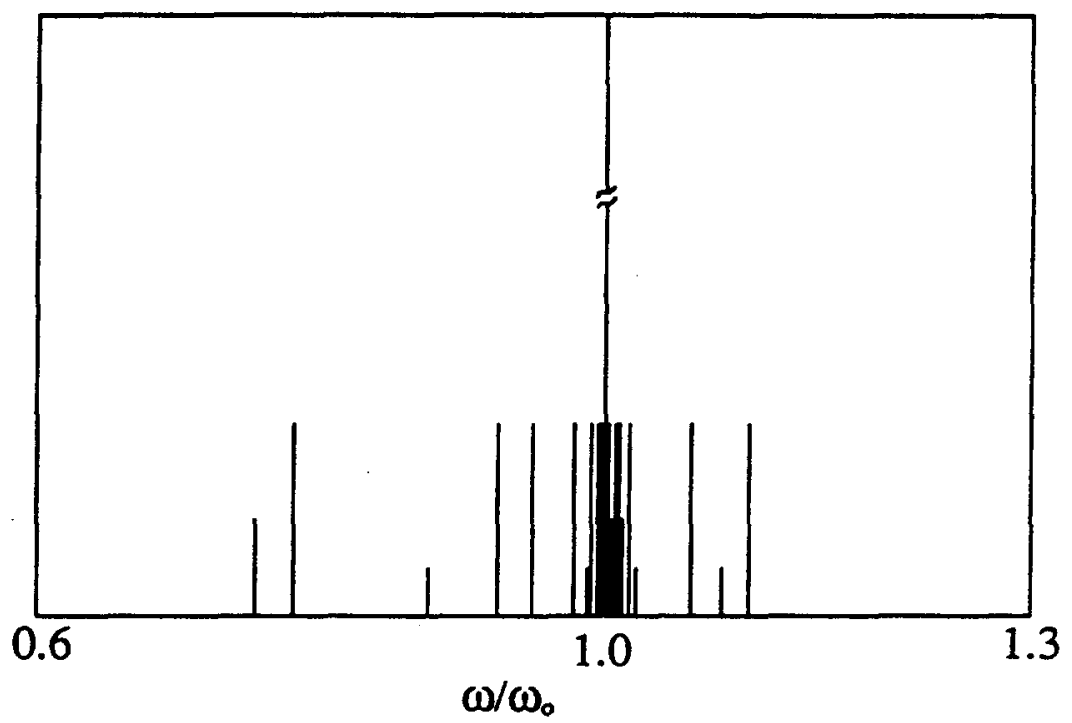

Figure 4. Calculated frequency distribution (arbitrary unit) of atoms in a $\Sigma=5$ twist boundary in gold at room temperanure based on the local-harmonic model.( $\omega_{0}$ is frequency of atoms in the perfect crystal). 